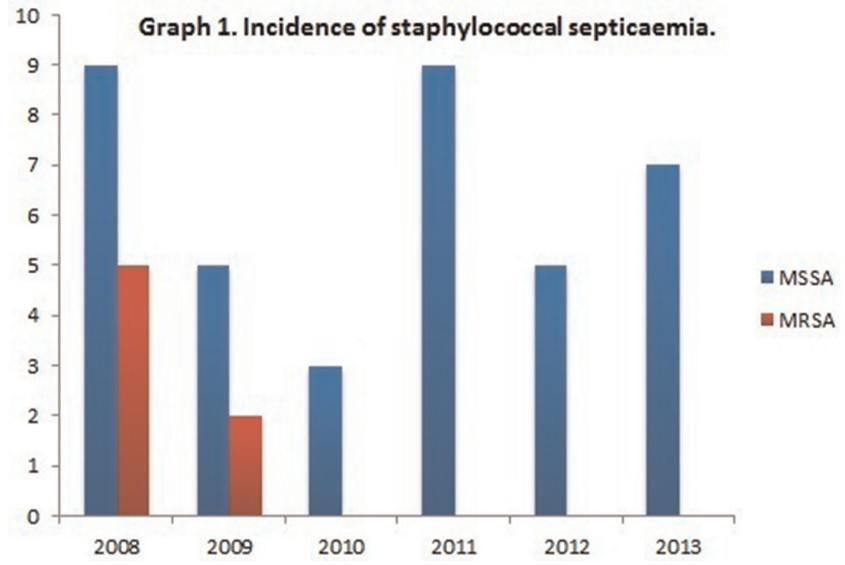

Abstract P0-0552 Figure 1

We describe the changing epidemiology of Staphylococcus aureus infections in NICU at Leeds over 2008-2013 using laboratory and clinical data.

Method Leeds neonatal service experienced an increased number of cases of Meticillin resistant Staphylococcus aureus (MRSA) colonisation and bacteraemia in 2008-2009. A series of infection control interventions were implemented stepwise including:

- asepsis training.

- weekly screening.

- adoption of the Saving Lives central venous catheter package,

- daily antiseptic skin washes in neonates $>28$ weeks.

- $2 \%$ Chlorhexidine for skin asepsis prior to invasive procedures.

Results There has been a noticeable success in reduction in MRSA infections and no bacteraemia has been reported since 2009 (Graph 1). A similar improvement has not been seen in Meticillin sensitive Staphylococcus aureus (MSSA) bacteraemia.

A retrospective review carried out to review MSSA bacteraemia since 2008: 71\% (27 of 38) cases were in neonates under 28 weeks, a vulnerable cohort currently excluded from daily skin washes.

Conclusions Given an association between MSSA colonisation and infection, further work should investigate infection control strategies that effectively target the highest risk groups and include active surveillance for MSSA and MRSA with subsequent decolonization.

\section{PO-0553 CONTRIBUTIONS OF CHANGES IN SERUM PROCALCITONIN CONCENTRATION IN THE TREATMENT OF SECONDARY SEPSIS IN NEWBORN}

\footnotetext{
${ }^{1}$ A Du Mesniladelée, ${ }^{1} \mathrm{~V}$ Champion, ${ }^{1} \mathrm{M}$ Lachtar, ${ }^{1} \mathrm{I}$ De Montgolfier, ${ }^{1} \mathrm{~F}$ Kieffer, ${ }^{2} \mathrm{D}$ Mitanchez. ${ }^{1}$ Neonatology, Hôpital Armand-Trousseau, Paris, France; ${ }^{2}$ Neonatology, Hôpital ArmandTrousseau Université Paris06, Paris, France
}

\subsection{6/archdischild-2014-307384.1195}

Background and aims Procalcitonin (PCT) is used in the early diagnosis of infections. Recently, PCT has been used in both adults and children as a guide to the duration of antibiotic treatment. The aims are to study the evolution of PCT during secondary sepsis in the newborn and to evaluate its ability to guide the duration of antibiotic treatment.

Patients and methods A prospective, observational study including all neonates hospitalised in a level II neonatal unit between
December 2011 and January 2013 with suspected infection after 5 days of life and serum PCT $>0.6 \mathrm{ng} / \mathrm{L}$. Serial PCT, CRP and blood culture survey was performed according to the usual protocol. Adapted antibiotherapy was administered for 10 days after the last positive blood culture.

Results 54 infective episodes were observed in 46 neonates, born at a mean term of 32 weeks (range: 26-40) and infected for a mean of 19 days (7-40). Staphylococci and gram-negative bacteria caused respectively $57 \%$ and $22 \%$ of infective episodes. At the time of clinical diagnosis (D0), 74\% of the PCT values and $81.5 \%$ of the CRP values were positive. Between D5 and D8,80\% of PCT measurements were negative $(<0.6 \mathrm{ng} / \mathrm{L})$ versus only $25 \%$ of CRP. On D8, $47.0 \%$ of CRP measurements were still positive. Had antibiotherapy been discontinued when PCT was $<0.6 \mathrm{ng} / \mathrm{ml}$, it would have been 5 days shorter.

Conclusion In newborn with secondary sepsis, serum PCT may help to reduce antibiotherapy duration and this should be examined in a controlled study.

\section{PO-0554 CREATING A NETWORK OF NEONATOLOGISTS, CHILD HEALTH RESEARCHERS, AND PUBLIC HEALTH SPECIALISTS TO STUDY NEONATAL INFECTIONS' RELATED MORTALITY AND MORBIDITIES IN EGYPT}

${ }^{1} \mathrm{M}$ Mohamed, ${ }^{2} \mathrm{~A}$ Roess, ${ }^{3} \mathrm{~A}$ Shaalan, ${ }^{1} \mathrm{H}$ Aly. ${ }^{1}$ Newborn Services, The George Washington University Medical Center, Washington DC, USA; ${ }^{2}$ Department of Global Health SPHHS, The George Washington University, Washington DC, USA; ${ }^{3}$ President, National Research Centre, Cairo, Egypt

\subsection{6/archdischild-2014-307384.1196}

Background Congenital and acquired infections not only play major rule in neonatal mortality in Egypt, but also lead to significant morbidities and lifelong handicaps among Egyptian newborns.

Objectives 1) Develop research agenda to study neonatal infections and their impact on neonatal mortality and morbidities in Egypt. 2) Facilitate the assembly of multidisciplinary, multicenter research teams to examine the most critical questions on this agenda.

Methods Through collaboration with Egyptian National Research Centre, authors organised a four-day workshop in Cairo, Egypt, to facilitate the interaction of Egyptian and US experts to achieve above objectives. Authors invited neonatologists, obstetricians, and child health researchers from academic, governmental, and private sectors across the country. A call for abstracts invited participants to share their clinical and laboratory research. Leading neonatologists were invited to share their field experiences and case studies. Authors distributed participants into five diverse teams. Workshop activities included panel presentations, open discussions, and team focused-interactions. While discussing current and emerging aspects of neonatal infections and their impact on neonatal mortality and morbidities in Egypt, each team was tasked to develop research questions to study causes, predisposing factors, and intervention or prevention methods. Findings of each team were verified through whole group revisions. Using epidemiologic and clinical criteria, participants used simple voting to create a consensus on the most critical issues. Each team concluded the workshop by designing a framework for a research project to examine one of these issues.

Results More than forty neonatologists, paediatricians, child health researchers, and obstetricians joined the workshop. Sixteen abstracts were selected for presentation. Eighteen guest 